4. N. Jacobson, Abstract derivations and Lie algebras, Trans. Amer. Math. Soc. 42 (1937), 206-224.

5. - - p-Algebras of exponent p, Bull. Amer. Math. Soc. 43 (1937), 667-670.

6. - Galois theory of purely inseparable fields of exponent one, Amer. J. Math. 46 (1944), 645-648.

7. - Lectures in abstract algebra, Vol. III, Van Nostrand, Princeton, N. J., 1964.

8. O. Zariski and P. Samuel, Commutative algebra, Van Nostrand, Princeton, N. J., 1958.

University of Pennsylvania

\title{
A SPARSE REGULAR SEQUENCE OF EXPONENTIALS CLOSED ON LARGE SETS
}

BY H. J. LANDAU

Communicated by P. D. Lax, March 30, 1964

Introduction. For a given sequence $\left\{\lambda_{k}\right\}$ of complex numbers, the problem of determining those intervals $I$ on which the exponentials $\left\{e^{i \lambda_{k} x}\right\}$ are complete in various function spaces has been extensively studied [3]. Since the problem is invariant under a translation of $I$, only the lengths of $I$ are involved, and attention has focused on the relation between these lengths and the density of the sequence $\left\{\lambda_{k}\right\}$. With the function space taken to be $L^{p}(I)$ for $1 \leqq p<\infty$, or $C(I)$, the continuous functions on $I$, the general character of the results has been that there exist sparse real sequences $\left(\lim r^{-1}\right.$ (the number of $\left.\left|\lambda_{k}\right|<r\right)=0$, for example) for which $I$ can be arbitrarily long [2], but all such sequences are nonuniformly distributed; when a sequence is sufficiently regular, in the sense that $\lambda_{k}$ is close enough to $k$, the length of $I$ cannot exceed $2 \pi$ [4, p. 210]. Most recently, in a complete solution which accounts for all these phenomena, Beurling and Malliavin have proved that the supremum of the lengths of $I$ is proportional to an appropriately defined density of $\left\{\lambda_{k}\right\}[1]$.

The purpose of this note is to show that the situation is quite different when the single interval $I$ is replaced by a union of intervals. Specifically, we will construct a real symmetric (or positive) sequence $\left\{\lambda_{k}\right\}$ arbitrarily close to the integers, for which the corresponding exponentials are complete in $C(S)$, where $S$ is any finite union of the intervals $|x-2 n \pi|<\pi-\delta$, with integer $n$ and $\delta>0$, and so has arbitrarily large measure. Thus, for sets $S$ more general than intervals, 
it would seem that no relation can be expected between measure of $S$ and density of $\left\{\lambda_{k}\right\}$.

Acknowledgment. I am very indebted to Professor Beurling for his interest and advice.

\section{Results.}

LEMMA 1. We may partition the positive integers into an infinite number of disjoint sequences $S_{r}=\left\{k_{n}^{(r)}\right\}_{n=1}^{\infty}, r=1,2, \cdots$, with the property that $\lim \sup n / k_{n}^{(r)}=1$ for each $r$.

Proof. We will define $S_{r}$ as the union $\bigcup_{i=1}^{\infty} \sigma_{i, r}$ of disjoint blocks $\sigma_{i, r}$ of consecutive integers. To define $\sigma_{i, r}$, we order the integer couples $(i, r)$ with $i, r \geqq 1$, by increasing values of $s=i+r$, and for same values of $s$, by increasing $i$. We let $\sigma_{1,1}=\{1\}$ and choose the remaining $\sigma_{i, r}$ consecutively in the order of the $(i, r)$, letting each $\sigma_{p, q}$ begin with the first integer not included in the previously defined $\sigma$; we pick $\sigma_{p, q}$ so long that if $N$ is the number of integers in $\sigma_{p, q}$, $k$ is the first of them, and $M$ is the total number of integers in the (already determined) $\sigma_{j, q}$ with $j<p$, then $(N+M) /(k+N-1)$ $>1-1 / p$. By this construction, whenever $k_{n}^{(r)}$ in $S_{r}$ coincides with the right-hand endpoint of a $\sigma_{i, r}$ we have $n / k_{n}^{(r)}>1-1 / i$, so that $\lim \sup n / k_{n}^{(r)}=1$ for each $r$. Finally, the $S_{r}$ are all disjoint and their union is all positive integers. Lemma 1 is established.

Leмma 2. With $\theta_{1}, \theta_{2}, \cdots$ real numbers, set $z_{k}=e^{i 2 \pi \theta k}$, and denote by $\Delta\left(\theta_{1}, \cdots, \theta_{n}\right)$ the determinant whose $2 j$ th row is $z_{j}^{n-1}, z_{j}^{n-2}, \cdots, z_{j}^{-n}$ and whose $(2 j-1)$ th row is $z_{j}^{-n+1}, z_{j}^{-n+2}, \cdots, z_{j}^{n}$, with $1 \leqq j \leqq n$. Then given $\epsilon>0$ we may choose $\theta_{1}, \theta_{2}, \cdots$ with $\left|\theta_{i}\right|<\epsilon$ so that, for all $n$, we have $\Delta\left(\theta_{1}, \cdots, \theta_{n}\right) \neq 0$.

Proof. The condition $\left|\theta_{i}\right|<\epsilon$ is equivalent to $z_{i} \in \gamma$, with $\gamma$ an appropriate arc of $|z|=1$. First, letting $z_{1}$ be any point of $\gamma$ other than $z=1$ ensures $\Delta\left(\theta_{1}\right) \neq 0$. Then we observe that $\Delta\left(\theta_{1}, \cdots, \theta_{n}\right)$ can be expanded as a polynomial in $z_{n}$ and $z_{n}^{-1}$, with leading coefficient $\Delta\left(\theta_{1}, \cdots, \theta_{n-1}\right)$. Assuming $z_{1}, \cdots, z_{n-1}$ have been chosen to satisfy the requirements of the lemma, this coefficient does not vanish, and so $\Delta\left(\theta_{1}, \cdots, \theta_{n}\right)$ considered as a function of $z_{n}$ is not identically zero; being analytic in $z_{n}$ it therefore cannot vanish everywhere for $z_{n}$ on $\gamma$. Thus we may find a point $e^{i 2 \pi \theta_{n}} \in \gamma$ such that when $z_{n}=e^{i 2 \pi \theta_{n}}$, $\Delta\left(\theta_{1}, \cdots, \theta_{n}\right) \neq 0$. By induction, Lemma 2 is established.

THEOREM. Given $\epsilon>0$, there exists a symmetric real sequence $\left\{\lambda_{k}\right\}_{-\infty}^{\infty}$ with $\left|\lambda_{k}-k\right|<\epsilon$ such that the functions $\left\{e^{i \lambda_{k} x}\right\}$ are complete in continu- 
ous functions on every finite union of the intervals $|x-2 n \pi|<\pi-\delta$, with integer $n$ and $\delta>0$.

Proof. We will partition the integers into disjoint subsets, shift each subset by a small amount, and let the sequence $\left\{\lambda_{k}\right\}$ consist of the points so obtained. Then we will show that completeness of the corresponding exponentials on unions of certain intervals is equivalent to completeness on a single interval of $\left\{e^{i k x}\right\}$, with $k$ in one subset, and thereby reduce the theorem to a classical result.

Let $S_{r}, r=1,2, \cdots$, be the disjoint subsets of the integers defined in Lemma 1, and let $S_{-r}=\left\{k \mid-k \in S_{r}\right\}$. Similarly, let $\theta_{r}, r=1,2, \cdots$, be the numbers constructed in Lemma 2 , and let $\theta_{-r}=-\theta_{r}$. Now for $k \in S_{r}, r= \pm 1, \pm 2, \cdots$, set $\lambda_{k}=k+\theta_{r}$, and $\lambda_{0}=0$. Then the sequence $\left\{\lambda_{k}\right\}_{-\infty}^{\infty}$ is symmetric and $\left|\lambda_{k}-k\right|<\epsilon$.

To prove the theorem we must show that given $N$ and $\delta>0$, the exponentials $\left\{e^{i \lambda_{k} x}\right\}$ are complete in $C(S)$, where $S=\bigcup_{n=-N+1}^{N} I_{n}$, and $I_{n}$ is the interval $|x-2 n \pi|<\pi-\delta$, or equivalently $[4$, p. 115] that any bounded measure supported on $S$ which annihilates these exponentials must vanish identically. Accordingly, let $\mu(x)$ be such a measure, and denote by $\mu_{n}(x-2 n \pi)$ the restriction of $\mu(x)$ to $I_{n}$. Then $\mu_{n}(x)$ is a bounded measure supported on $I_{0}$, and

$$
\mu(x)=\sum_{n=-N+1}^{N} \mu_{n}(x-2 n \pi) .
$$

Now by a change of variable,

$$
\int_{S} e^{i \lambda_{k} x} d \mu(x)=\sum_{n=-N+1}^{N} e^{i \lambda_{k} 2 n \pi} \int_{I_{0}} e^{i \lambda_{k} x} d \mu_{n}(x),
$$

and if $k \in S_{r}, e^{i \lambda_{k} 2 n \pi}=e^{i \theta_{r} 2 n \pi}$ and does not depend on $k$. Thus if $\mu(x)$ annihilates the exponentials $\left\{e^{i \lambda_{k} x}\right\}$ for $k \in S_{r}$, so does

$$
\sum_{n=-N+1}^{N} e^{i \theta_{r} 2 n \pi} \mu_{n}(x)
$$

which is a bounded measure supported on the single interval $I_{0}$.

We now invoke a known result [3, p. 13]: since by Lemma 1, $\lim \sup n / k_{n}=1$ in each $S_{r}$, the set $S_{r}$ has Polya density 1 , and so the exponentials $\left\{e^{i k x}\right\}$ for $k \in S_{r}$ are complete in continuous functions on any interval of length less than $2 \pi$, in particular on $I_{0}$. By definition of the set $\left\{\lambda_{k}\right\}$ for $k \in S_{r}$ as a translate of $S_{r}$, the same is true of the exponentials $\left\{e^{i \lambda_{k} x}\right\}, k \in S_{r}$, and consequently the measure (2) on $I_{0}$ which annihilates them must vanish identically. We conclude 


$$
\sum_{n=-N+1}^{N} e^{i \theta_{r} 2 n \pi} \mu_{n}(x) \equiv 0, \quad x \in I_{0},
$$

for each $r$. Writing (3) with $r= \pm 1, \cdots, \pm N$ yields a system of $2 N$ linear equations for the $2 N$ measures $\mu_{n}(x),-N+1 \leqq n \leqq N$, whose determinant is precisely $\Delta\left(\theta_{1}, \cdots, \theta_{N}\right)$ and so, by Lemma 2 , does not vanish. Thus the only solution to this system is $\mu_{n}(x) \equiv 0,-N+1$ $\leqq n \leqq N$, whence $\mu(x) \equiv 0$ by (1). This completes the proof of the theorem.

Remarks. 1. By an obvious modification of the proof, the exponentials $\left\{e^{i \lambda_{k} x}\right\}$ with $k \in S_{r}$ for $r>0$ have the same completeness property.

2. We may give a constructive proof of the theorem along the same lines. Shifting each $I_{n}$ to $I_{0}$ transforms the problems of approximating a continuous function on $S$ by linear combinations of the exponentials $\left\{e^{i \lambda_{k} x}\right\}$ into that of solving a system of linear equations on $I_{0}$ with nonzero determinant, and thereby again reduces matters to approximating on $I_{0}$ by linear combinations of $\left\{e^{i k x}\right\}$ for $k \in S_{r}$.

\section{BIBLIOGRAPHY}

1. A. Beurling and P. Malliavin, On the closure of a sequence of exponentials on a segment, Multigraphed lectures, Summer Institute on Harmonic Analysis, Stanford University, Stanford, Calif., 1961.

2. J. P. Kahane, Sur la totalite des suites d'exponentielles imaginaires, Ann. Inst. Fourier (Grenoble) 8 (1958), 273-275.

3. N. Levinson, Gap and density theorems, Amer. Math. Soc. Colloq. Publ. Vol. 26, Amer. Math. Soc., Providence, R. I., 1940.

4. F. Riesz and B. Sz-Nagy, Functional analysis, Ungar, New York, 1955.

Bell Telephone laboratories 